\title{
Moving Surface Actuation, Effects of Frequency-based Shear Layer Excitation on the Response of a Bluff Body Wake
}

\author{
M. R. Singbeil ${ }^{1 *}$, C. Ghiroaga ${ }^{1}$, C. Morton ${ }^{1}$, R. J. Martinuzzi ${ }^{1}$ \\ ${ }^{1}$ University of Calgary, Mechanical and Manufacturing Engineering Department, Calgary, Canada \\ *mrsingbe@ucalgary.ca
}

\begin{abstract}
The effect of actuation frequency, using moving surface actuation, is investigated for a square cylinder bluff body wake. Pressure sensor data are used to optimize actuation characteristics through the implementation of an NSGA-II evolutionary algorithm. Velocity field data are obtained using Particle Image Velocimetry (PIV) for baseline and optimized actuation cases. A Proper Orthogonal Decomposition (POD) analysis shows that the vortex shedding frequency shifts between frequencies associated with the actuation, moving between regions of lock-on and quasi-periodicity. Additionally, the POD shows that the energy contained in the coherent shedding motion is reduced through actuation, while the total energy in the velocity field stays relatively constant. A reconstruction of the first 10 POD modes indicates that the coherent contribution to the Reynolds stresses significantly decreases compared to the non-actuated case. The mechanism for drag reduction is investigated using the shed circulation flux and Kochin's drag formulation model. The drag obtained using PIV measurements and Kochin's formulation is consistent with trends observed for the base pressure as a function of actuation frequency.
\end{abstract}

\section{Introduction}

Vortex shedding is a common phenomenon caused by the interaction of two opposing shear layers in the wake of a bluff body. These interactions give rise to coupled instabilities leading to the quasi-periodic shedding of vortices (Williamson, 1996). These vortices induce fluctuating loads which are major contributors to drag forces acting on high-speed vehicles and cyclic loading associated with flow-induced vibrations of structures. These forces are proportional to the strength of the shed vortices (i.e. circulation) and the rate at which they convect (Kochin, 1964); both of which depend on the rate at which circulation is generated and advected from the body's surface. Due to the importance of circulation on the shedding process, actuation methods for manipulating the vorticity flux is vital for its control. Hence, the work focuses on how frequency-modulated moving surface actuation affects the formation and shedding of vortices to control wake dynamics.

Shear layer excitation has potential to manipulate the circulation generation and transport to exploit flow instabilities to alter the shedding process (Xu et al., 2017). The square cross-sectional prism was selected to isolate the influence of actuation by fixing the separation point and avoiding downstream shear layer reattachment. Separation at the leading edge reduces the Reynolds number sensitivity of aerodynamic characteristics (Okajima, 1982) such as drag which is strongly correlated to the shed circulation flux in the wake (Kochin, 1964). Actuating at the inception of shear layer separation is achieved with a symmetric pair of moving surface actuators (rotating cylinders) at the leading edges. Previous work with moving surface actuation, such as Munshi et al. (1997), note very high surface velocity to free-stream ratios $\left(v_{s} / U_{\infty}\right)$ on the order of 3 times to achieve authority on the wake. These studies focused on boundary layer control through the addition of significant momentum resulting in delayed separation. In contrast, frequency based actuation methods exploit the frequency dependent nature of a bluff body wake achieving authority with less input energy (Wu et al. 2007). The rotating cylinders experience a whirling motion which introduces a forcing frequency and cascading harmonics at integer multiples of the rate of rotation $\left(f_{w}=N / 60\right)$ where $N$ is the revolutions per minute, RPM, of the motor (Baek and Sung, 2000). 
In this study, the effects of frequency-based moving surface actuation on the base pressure and wake dynamics are investigated. Pressure on the obstacle's surface is complemented by velocity data obtained using PIV. Results are investigated using Fourier analysis and POD.

\section{Methodology}

The experiments were performed in a small-scale suction type wind tunnel shown in Figure 1. Air is drawn through four conditioning grids at the inlet before undergoing a 9:1 contraction down to the square test section with a wetted cross-sectional area of $203.2 \mathrm{~mm} \times 203.2 \mathrm{~mm}$ and is $508.0 \mathrm{~mm}$ long. The obstacle is a square cross-section prism with a characteristic length $D=25.4 \mathrm{~mm}(\mathrm{Fig} 2)$. The obstacle is mounted wall to wall with an aspect ratio of 8:1 and results in a blockage of $12.5 \%$ when installed $127.0 \mathrm{~mm}$ downstream of the test section inlet.

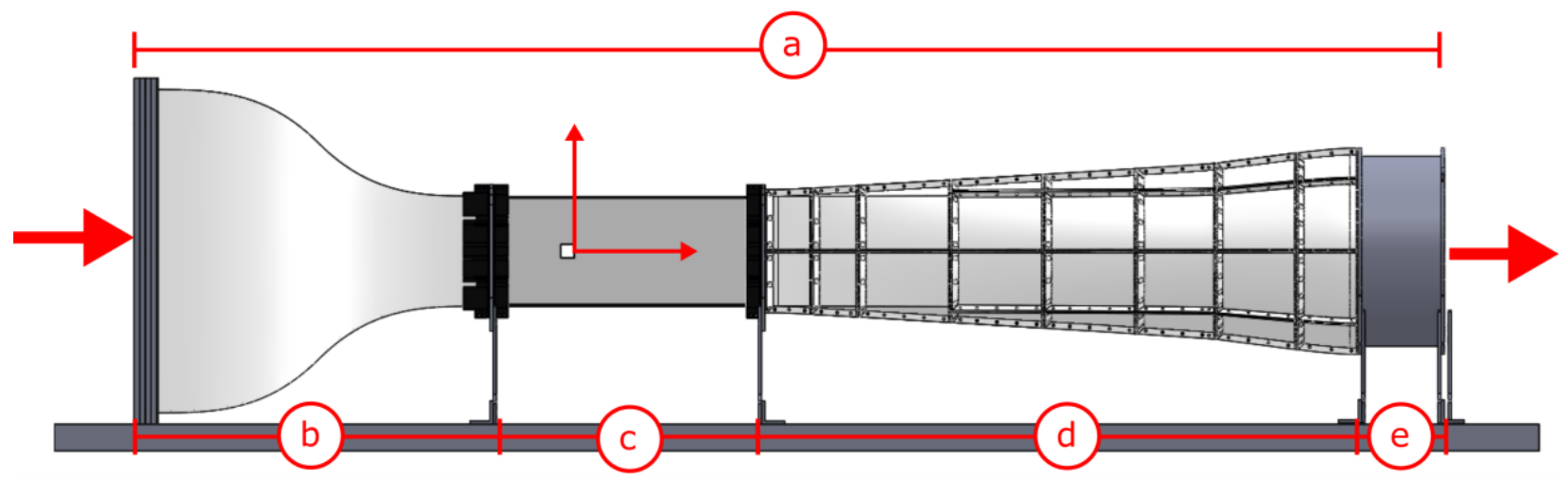

Figure 1: Side View of Wind Tunnel Facility

The actuators are two $10 \mathrm{~mm}$-diameter rotating cylinders driven via two brushless DC servo-motors mounted external to the test section. Six differential pressure transducers are used to obtain pressure at locations $P_{1}$ to $P_{6}$ indicated in Fig. 2. On the top/bottom surfaces, transducers $P_{1}$ and $P_{2}$ are 1-inch water column (All Sensors D-4V) and on the leeward face $P_{3}$ to $P_{6}$ are 5-inch (All Sensors Dx-4v Mini) transducers.

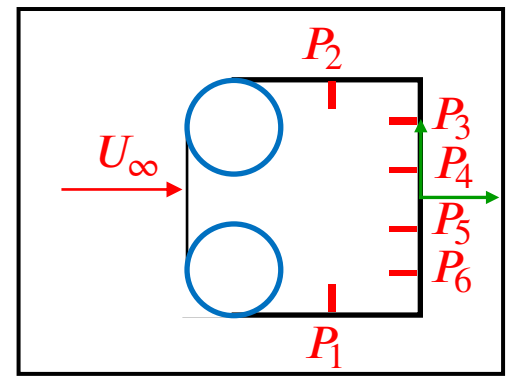

Figure 2: Obstacle with surface mounted pressure taps and origin of the PIV domain on the leeward surface

A modified multi-objective NSGA-II sorting algorithm (Deb et al., 2002) was used to explore the space of actuation parameters and reduce the dimensionality of the search space. Multiple trials were performed first optimizing the direction and rate of symmetric cylinder rotation $(r(t)=D \times N)$, then introducing sinusoidal modulation of the motor control signal $(r(t)=A \sin (2 \pi f t)+N)$, followed by asymmetric actuation with a phase difference between motors $(r(t)=A \sin (2 \pi f t+\phi)+N)$.

Over all trials the rate of rotation was limited to $0<N<3000 \mathrm{RPM}$, the oscillation amplitude varied between 5 and 50 RPM with a frequency of 0 to $4 \mathrm{~Hz}$, and the phase difference ranging from 0 to $\pi$ radians. These limits were chosen due to the stability margins of the servo-motor apparatus. The fitness functions prescribed to the genetic algorithm were set to maximize the average leeward face base pressure $\left(\overline{C_{p b}}=\right.$ $\left.\frac{1}{4}\left[\overline{C_{P 3}}+\overline{C_{P 4}}+\overline{C_{P 5}}+\overline{C_{P 6}}\right]\right)$, and minimize the RMS of the top and bottom pressure fluctuations $\left(C_{p l}^{\prime}=C_{P 1}^{\prime}+\right.$ 
$C_{P 2}^{\prime}$ ) in order to maintain diversity of solutions. The non-dominating set of individuals within the final pareto front for each trial indicated that the base pressure was highest with symmetric actuation at a constant RPM in the positive sense of rotation with respect to the flow; thus reducing the search to a single parameter, $N$. As such, a grid search was performed gathering pressure data from 0 to 3000 RPM in increments of 5 RPM.

Planar PIV was performed to gather instantaneous two-dimensional velocity data in the $\mathrm{x}$ and $\mathrm{y}$ directions. $N=0,2000,2180,2200$, and 3000 RPM were selected in order to investigate lock-on, desynchronization, and the quasi-periodic states. A single cavity Photronics Industries laser with a wavelength of 527 $\mathrm{nm}$ was operated in dual-pulse mode. Each pulse of the laser was synchronized with a Phantom Miro Lab 340 high-speed camera using a LaVision High-Speed Controller. The laser beam was reflected through a cylindrical lens to illuminate the area six diameters downstream at the mid span of the obstacle. The inlet was seeded with atomized olive oil particles which are entrained uniformly into the test-section.

An area of $6 \mathrm{D} \times 6 \mathrm{D}$ was captured immediately downstream of the obstacle. A sampling rate of 600 frames per second was chosen to capture 10 instantaneous velocity fields per shedding cycle. La Vision DaVis 8.3 image processing software was used to process the raw images into instantaneous two-dimensional velocity data. The fluctuating image field is processed with a multi-grid window deforming algorithm to produce the velocity field for each image pair. The first pass used a window size of $32 \times 32$ pixels and an overlap of $50 \%$ whereas the final pass used a window size of $16 \times 16$ pixels with no overlap to result in equal resolution of both the spatial and vector fields. The resulting vector field had a resolution of $0.0638 \mathrm{D}$ in both $\mathrm{x}$ and $\mathrm{y}$ directions.

Fourier analysis of the pressure data is performed to investigate the wake response to the actuation (Fig. 3b). From the velocity field data gathered a POD analysis is performed to isolate changes in the spectra associated the vortex shedding, redistribution of Total Kinetic Energy (TKE) amongst the modes, and reductions in the Reynolds stress associated with coherent fluctuations. The average circulation flux in the shear layer and shed vortices is then extracted using a phase average and formulation for the mechanism of drag reduction presented.

\section{Results}

\subsection{Pressure Measurements}

Over the range of 0 to 3000 RPM, the pressure coefficient on the obstacle base was found to increase from -1.27 to -1.04 with regions of significant change followed by plateaus, indicated with red lines in Fig. 3 a.
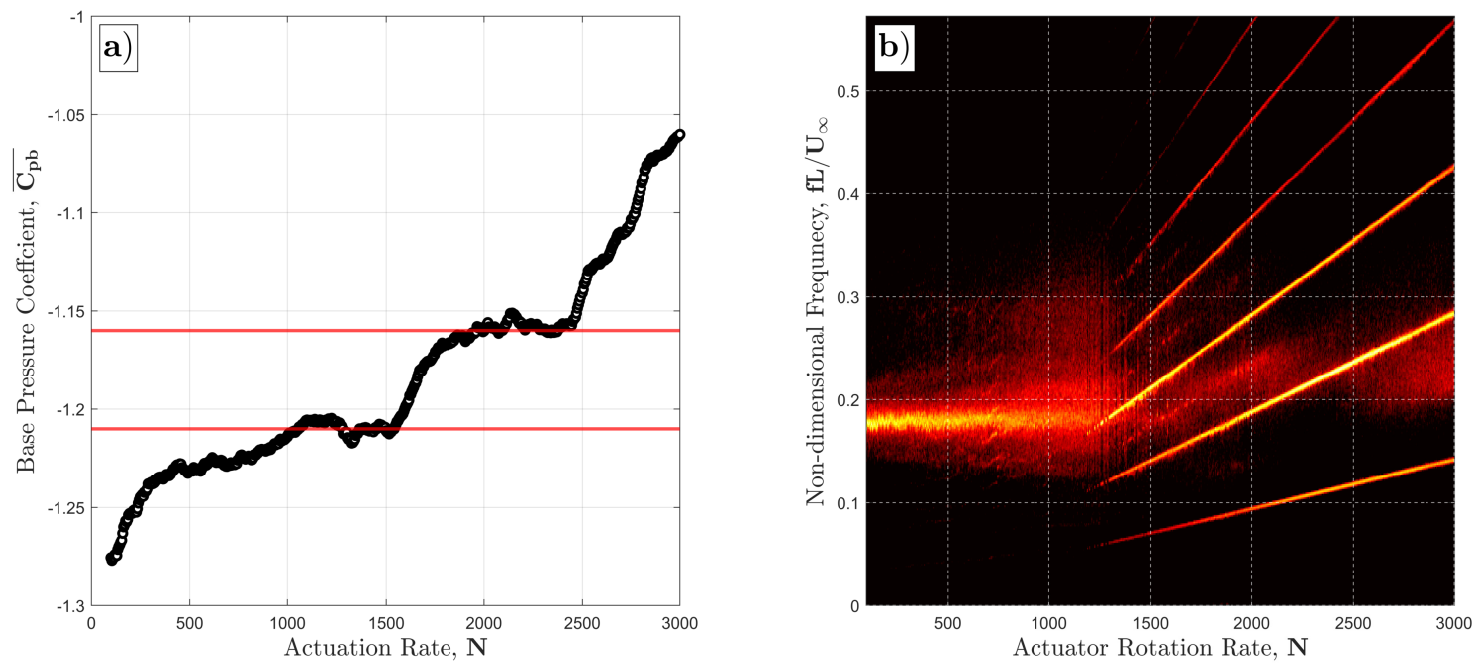

Figure 3: a) The base pressure coefficient computed from the 4 pressure taps on rear face of the obstacle versus the actuation rate, $N$; b) Flooded iso-contours of the PSDF computed from signal $X=C_{p 3}^{\prime}-C_{p 6}^{\prime}$ at each actuation rate, $N$.

The iso-contours of the Power Spectral Density Function (PSDF), in Fig. 3p, show the energy associated with frequencies in the wake. Below $N \approx 1250 \mathrm{RPM}$, the highest energy is concentrated near the baseline 
shedding frequency $(S t=0.184)$. Surpassing $1250 \mathrm{RPM}$, concentrations of energy (or rays) form, corresponding to the harmonics of the actuation frequency $\left(f_{w}\right)$. Changes in the intensity of the PSDF suggest regions of vortex lock-on, which shift with respect to these harmonics. In Fig. 3 a the first plateau in $\overline{C_{p b}}$ occurs from $\approx 1250$ to $1500 \mathrm{RPM}$ as the ray associated with the third harmonic $\left(3 f_{w}\right)$ first appears near the unactuated shedding frequency. From 1500 to 2000 RPM a cloud of dispersed spectral energy is observed between the second and third harmonic rays. From 2000 to approximately 2400 RPM a second plateau in $\overline{C_{p b}}$ can be seen in Fig. 3a, while the results in Fig. 3b suggest that the shedding frequency locks-on to the second harmonic of actuation $\left(2 f_{w}\right)$ (i.e., the cloud of dispersed spectral energy between $2 f_{w}$ and $3 f_{w}$ vanishes while the second harmonic appears near the unactuated shedding frequency). Continuing past 2500 RPM the base pressure continues to trend upward as the shedding frequency desynchronizes from the second harmonic ray. The results indicate that the plateaus in $\overline{C_{p b}}$ are linked to the state of the wake as the shedding frequency is either modulated by the nearby harmonics or experiences lock-on as the rays cross the unactuated shedding band.

\subsection{POD Analysis and Coherent contributions to the Reynolds Stress}

Performing a POD analysis, the distinct shedding frequencies for each PIV data set are extracted from the spectra of the first temporal coefficient (Fig. 4) and gathered in Table 1. From 2000 to 2200 RPM the spectral peak associated with the shedding frequency is seen to coincide with the second harmonic of the actuation further supporting lock-on over this range. At 2350 RPM the shedding frequency begins to desynchronize from the actuation as the harmonic peak approaches the edge of the unactuated shedding band and by 3000 RPM is fully desynchronized experiencing modulation by the neighbouring actuation harmonics and in a state of quasi-periodicity as outlined by Baek and Sung (2000). These regimes match the behaviour seen in Fig. 3 b supporting that the distinct changes in the base pressure shown in Fig. 3 are associated with the vortex shedding frequency transitioning between states of lock-on and quasi-periodicity with respect to the actuation harmonics.

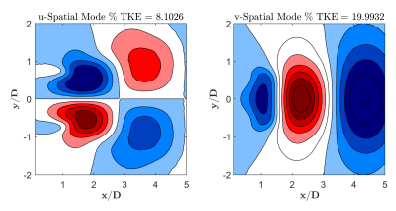

(a) $N=0 ; v_{s} / U_{\infty}=0$
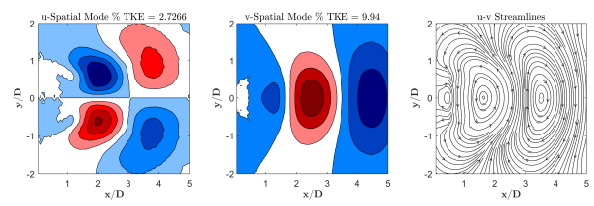

(c) $N=2180 ; v_{s} / U_{\infty}=0.130$
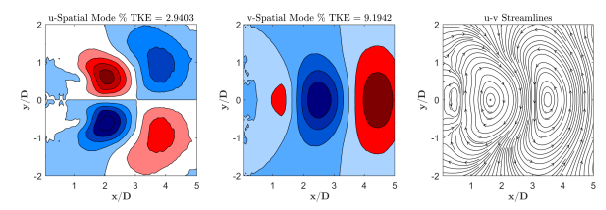

(e) $N=2350 ; v_{s} / U_{\infty}=0.140$
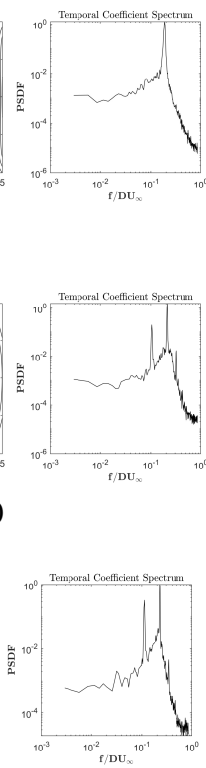
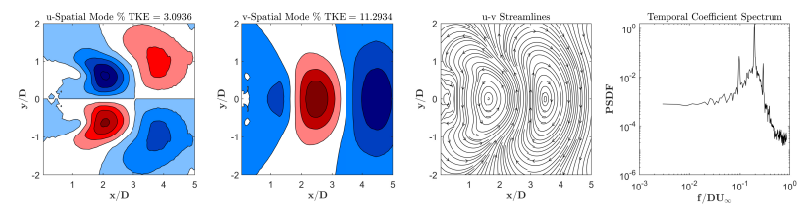

(b) $N=2000 ; v_{s} / U_{\infty}=0.119$
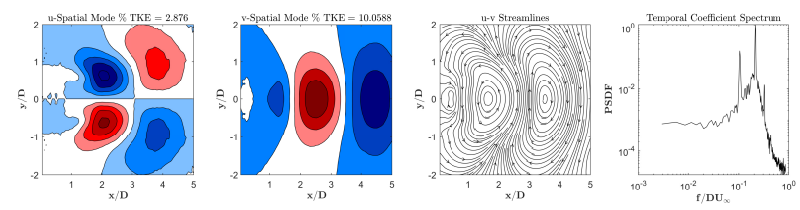

(d) $N=2200 ; v_{s} / U_{\infty}=0.131$
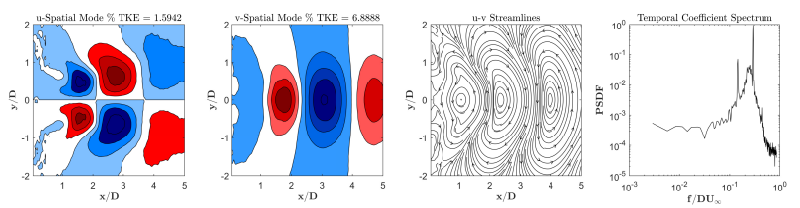

(f) $N=3000 ; v_{s} / U_{\infty}=0.178$

Figure 4: Spatial modes $\left(\Psi_{1}\right)$ with the spectra of their respective temporal coefficients $\left(a_{1}\right)$ for each PIV case 


\begin{tabular}{||c|c|c|c||}
\hline$N$ & $f_{w}$ & $2 f_{w}$ & $f_{v s}$ \\
\hline \hline 0 & 0 & 0 & 0.188 \\
\hline 2000 & 0.0097 & 0.194 & 0.194 \\
\hline 2180 & 0.1055 & 0.211 & 0.211 \\
\hline 2200 & 0.1060 & 0.212 & 0.212 \\
\hline 2350 & 0.1130 & 0.226 & 0.208 \\
\hline 3000 & 0.1445 & 0.289 & 0.222 \\
\hline \hline
\end{tabular}

Table 1: Non-dimensional frequency $\left(f^{*}=f D / U_{\infty}\right)$ of the first and second harmonics of the actuation along with the shedding frequency from each PIV case

Figure 5 shows the distribution of TKE among the first 100 POD modes for each PIV set. A reconstruction from the first 10 modes is performed in order to isolate changes in the fluctuating velocity field associated with coherent motions. This reconstruction captures approximately $62 \%$ of the TKE in the baseline case (Fig. 5b) and resolves $90 \%$ of the $\overline{u^{\prime} v^{\prime}}$ field with only minor discrepancies in the shear layer due to incoherent fluctuations.

From the reconstructed field, iso-contours of the Reynolds stress with overlaid mean streamlines are presented in Fig. 6 for each PIV case. From the baseline to the actuated cases, the streamwise position of $\max \left[\overline{u^{\prime 2}}\right]$ is seen to shift upstream along with the recirculation foci. This shift increases the relative distance from the foci to the maxima for both $\overline{u^{\prime} v^{\prime}}$ and $\overline{v^{\prime 2}}$ indicating that $v^{\prime}$ fluctuations occur further downstream from the recirculation nodes. This behaviour along with the vanishing concentrations of $u^{\prime} v^{\prime}$ within the recirulation bubble indicate a change in the vortex formation. As $N$ increases, the recirculation length $\left(L_{r}\right)$ is seen to decrease supporting a change in the mean wake structure. The distribution of Reynolds stress over the domain shows a reduction in strength of both the fluctuating velocity field within the shear layer $\left(\overline{u^{\prime 2}}\right)$ as well as downstream of the recirculation length $\left(\overline{v^{\prime 2}}\right)$. These reductions indicate a reduced energy associated with both the formation and shedding of vortices, supporting a link with the observed trend in $\overline{C_{p b}}$.
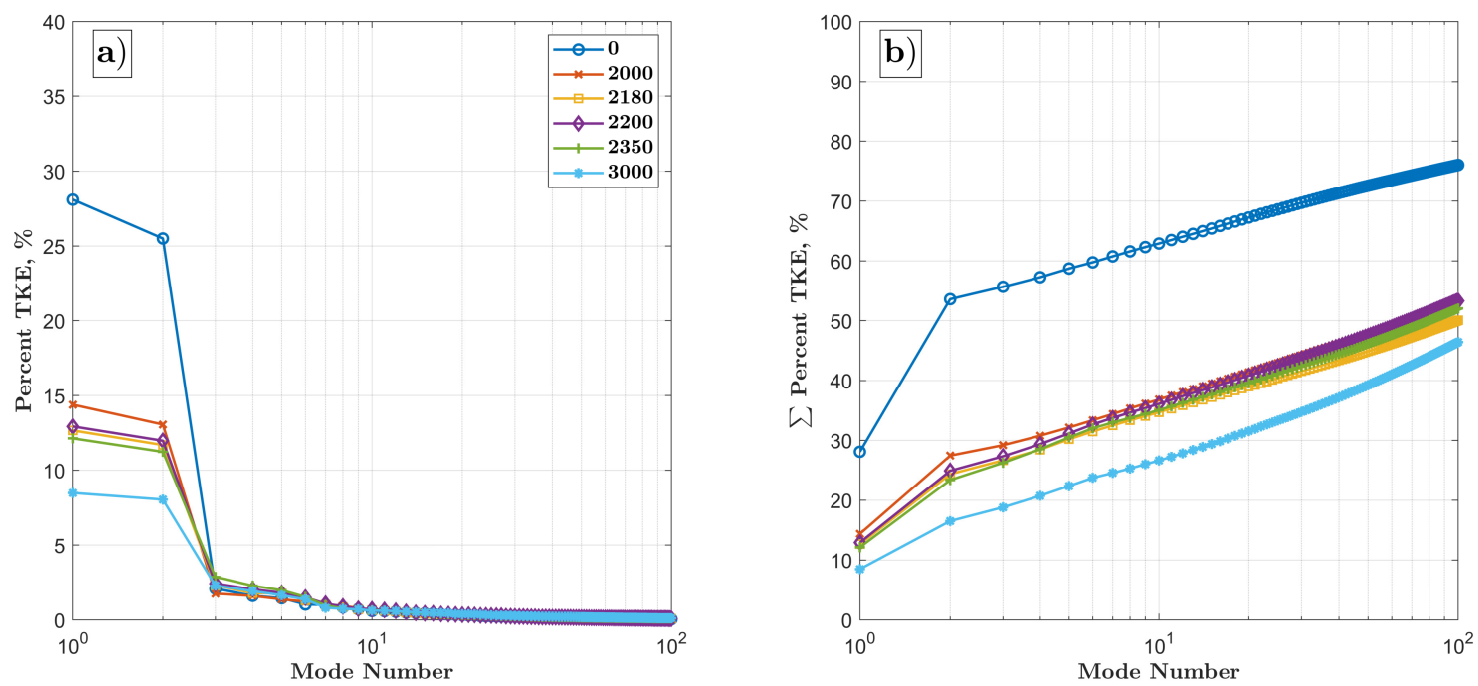

Figure 5: a) Percentage of the TKE captured for each of the first 100 modes b) cumulative sum of the modal energies for the first 100 modes 


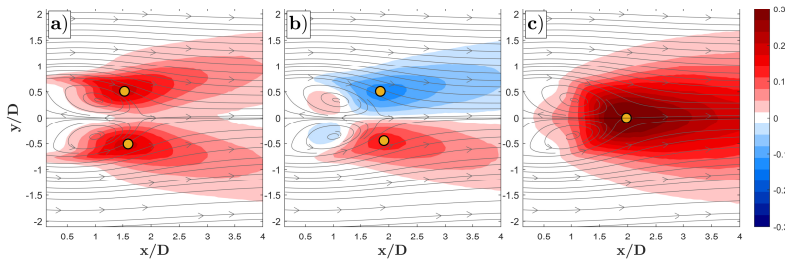

(a) $N=0 ; v_{s} / U_{\infty}=0$

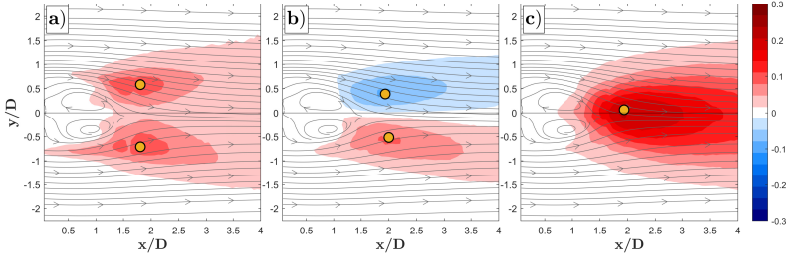

(c) $N=2180 ; v_{s} / U_{\infty}=0.130$

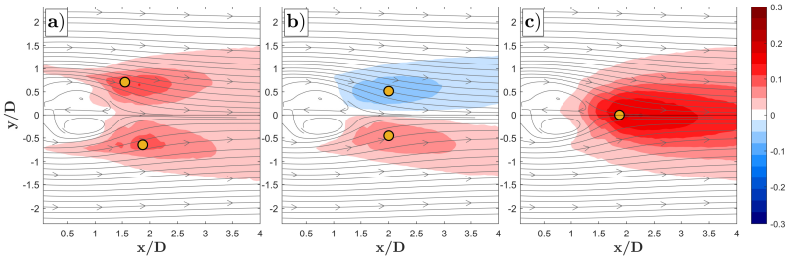

(e) $N=2350 ; v_{s} / U_{\infty}=0.140$

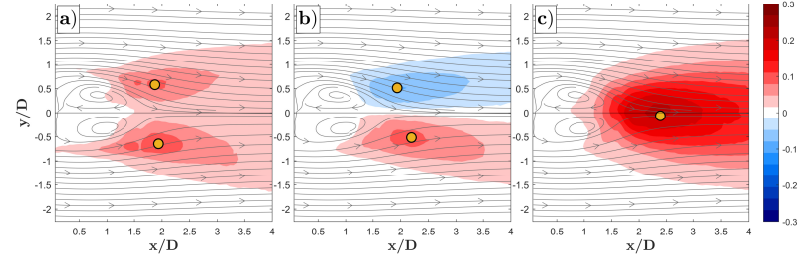

(b) $N=2000 ; v_{s} / U_{\infty}=0.119$

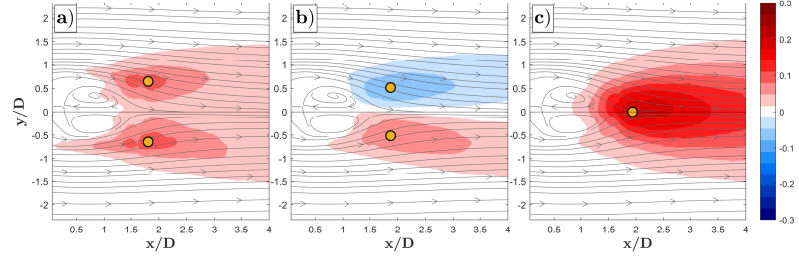

(d) $N=2200 ; v_{s} / U_{\infty}=0.131$

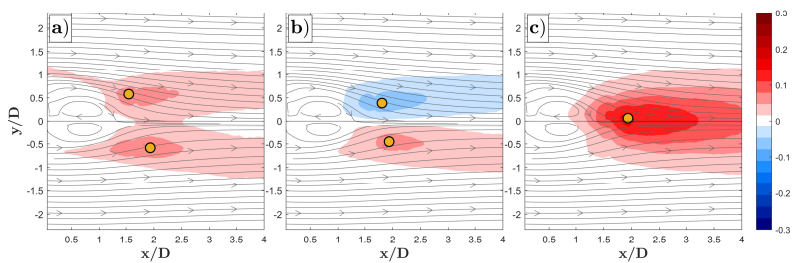

(f) $N=3000 ; v_{s} / U_{\infty}=0.178$

Figure 6: Iso-contours of Reynolds stress components $\overline{u_{c}^{\prime 2}}, \overline{u_{c}^{\prime} v_{c}^{\prime}}$, and $\overline{v_{c}^{\prime 2}}$ with overlaid mean streamlines in plots $\mathrm{a}, \mathrm{b}$, and $\mathrm{c}$ from the 8 mode reconstructed velocity field for each PIV case

To investigate changes of the Reynolds stresses with $N$, the maximum values for each component of the Reynolds stress are compared in Figure 7 for both the RANS and reconstructed fields. Matching the trends observed in Figure 6, reductions are noted in both $u^{\prime}$ and $v^{\prime}$ from the baseline to the actuated cases. However, as $N$ increases, reductions are observed in $\overline{u^{\prime} v^{\prime}}$ and $\overline{v^{\prime 2}}$ indicating a less energy associated with the shed vortices.
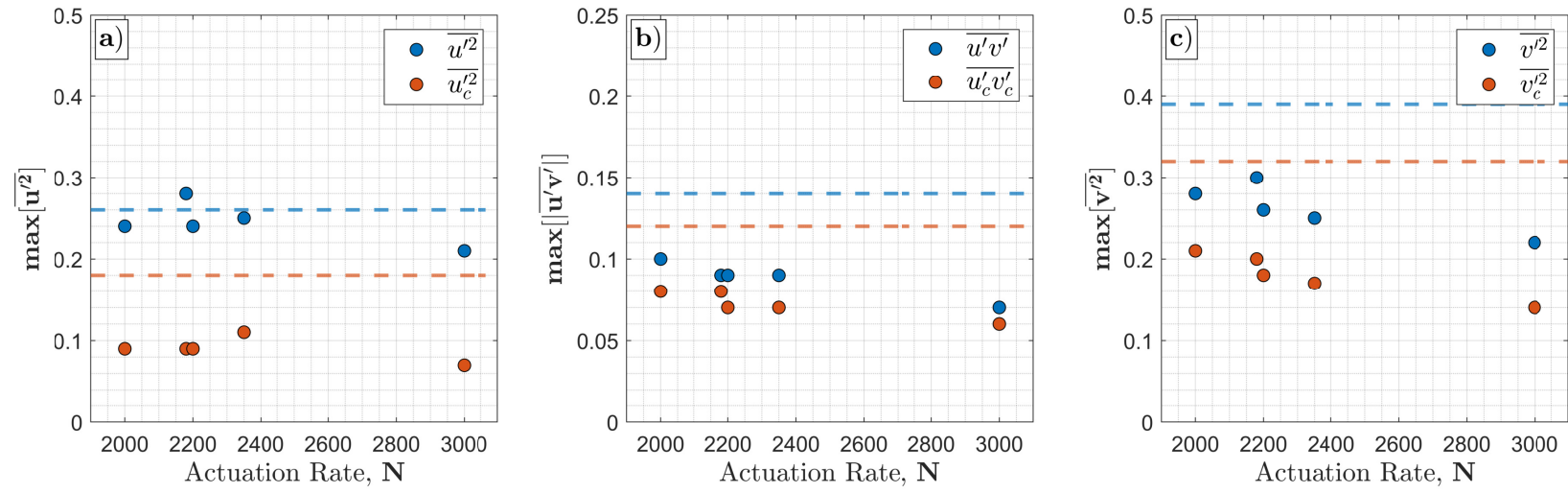

Figure 7: Maximum coherent contribution to the Reynolds stress from the reconstructed field $\overline{u_{c}^{\prime 2}}, \overline{u_{c}^{\prime} v_{c}^{\prime}}$, and $\overline{v_{c}^{\prime 2}}$ (shown in red) and maximum of the Reynolds stress $\overline{u^{\prime 2}}, \overline{u^{\prime} v^{\prime}}$, and $\overline{v^{\prime 2}}$ (in blue) with respect to the rate of rotation, $N$. Values for the baseline $(N=0)$ case are shown as dashed lines.

From the POD analysis reductions in the TKE associated with the first harmonic pair are observed. Comparing the baseline and first actuated case $(N=2000)$ a reduction from 54 to $16 \%$ is seen in the first 
harmonic pair in Fig. 5. In order to quantify the significance of reductions in TKE associated with the vortex shedding, the TKE within the domain and TKE from the first harmonic pair are plotted with respect to $N$ in Fig. 8. As the rate of actuation increases, the TKE associated with the first harmonic pair is reduced by a greater percent than changes in the TKE of the domain. It can be inferred that the decrease in fluctuations seen in the Reynolds stress are therefore likely associated with energy moving away from the shedding motion and exciting other coherent motions (or modes).
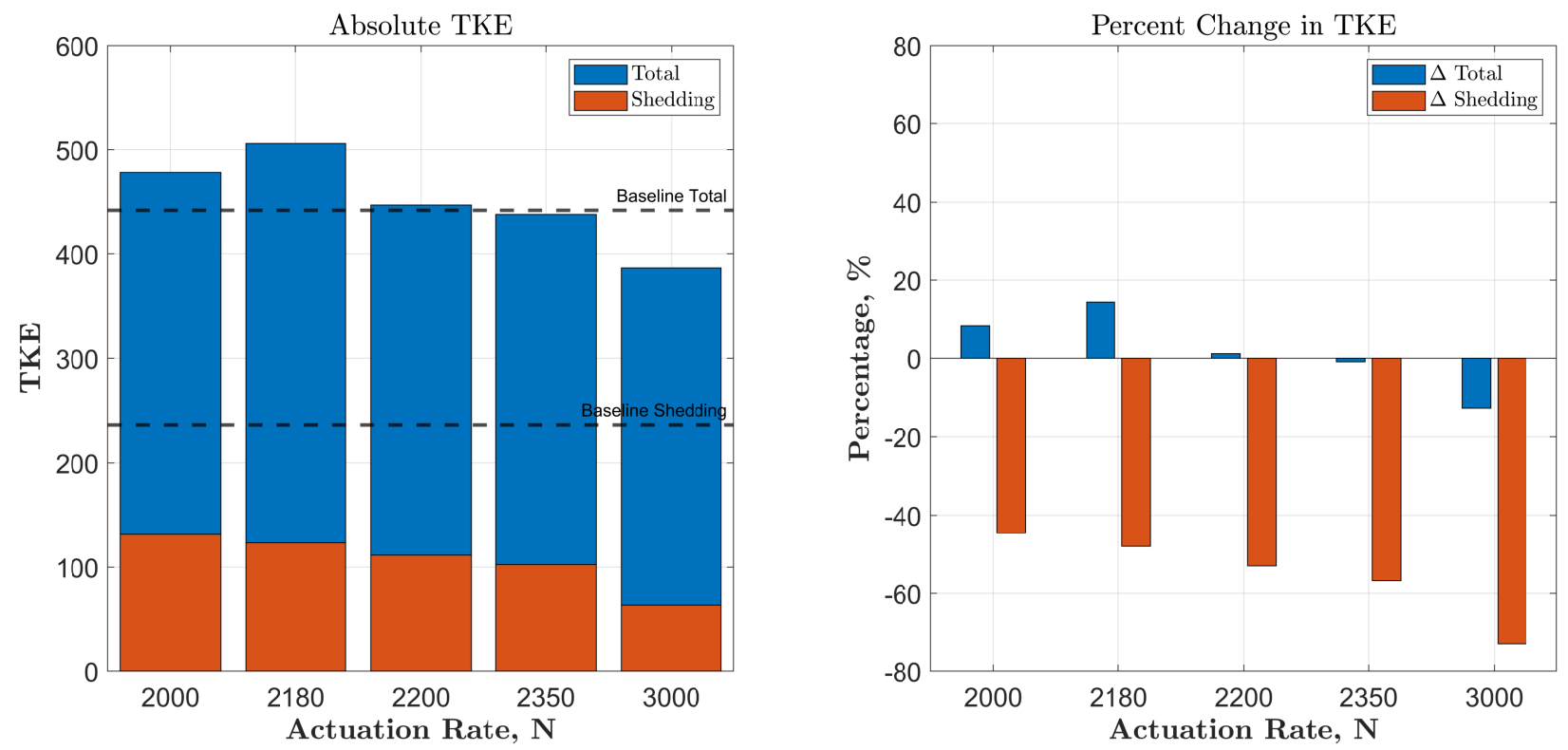

Figure 8: a) Absolute TKE captured the PIV domain and the shedding pair for each case b) Percent change in TKE from baseline values for the total, shedding pair computed from $\left[\frac{\text { case-baseline }}{\text { baseline }} \times 100 \%\right]$

\subsection{Circulation Flux in the Wake}

To investigate how circulation in the wake varies with the observed changes in the Reynolds stress both the flux along the shear layer and within the shed vortices are estimated. The circulation flux along the shear layer may be computed by finding the streamwise maximum of $d \Gamma_{s l}(x) / d t=\int_{y \mid U_{\min }}^{y \mid U_{\max }} U_{i} \Omega_{i} d y$ for each case and are shown as functions of $N$ in Figure 9. Next, a conditional average is performed using the phase computed from the temporal coefficients from the POD $a_{1}$ and $a_{2}\left(\theta=\tan ^{-1}\left(\frac{a 1}{\sqrt{2 \lambda_{1}}} / \frac{a 2}{\sqrt{2 \lambda_{2}}}\right)\right)$. The vortex core is then identified (as a connected regions of $Q>0$ ) and the contained vorticity integrated for each frame. This value is an estimate of the circulation flux contained in a shed vortex (severed from the shear layer) which is multiplied by the respective shedding frequency and plotted in Figure 9 

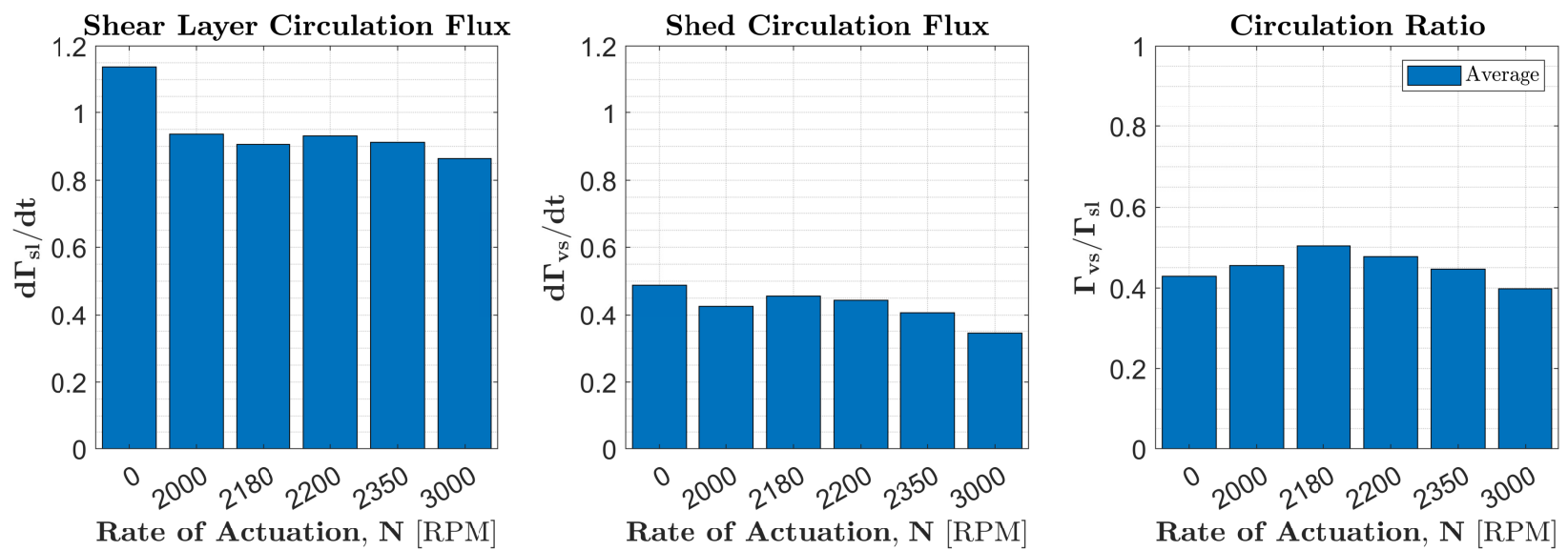

Figure 9: Circulations flux in the shear layer, circulation flux in the shed vortices, and the ratio of these two quantities for each PIV case.

From Fig. 9 the circulation flux in the shear layer is seen to drop by roughly $18 \%$ from $N=0$ to 2000 RPM, minimally vary in the range of lock-on from 2000 to 2200 , and further decrease from 2350 to 3000 RPM aligning with the reductions in $u^{\prime}$ and $v^{\prime}$ noted in Fig. 7 while the flux in the shed vorticies is only seen to trend downward slightly over the range. The ratio of circulation flux in the shed vortices to that in the shear layer is then computed to quantify if the circulation supplied to the domain and that captured by the shed vortices is changing as a result of the actuation. From the values in Fig. 9 no clear trend is observed as the changes in $\Gamma_{v s} / \Gamma_{s l}$ do not match the increase in $\overline{C_{p b}}$. Thus, it is speculated that the actuation is not just reducing the energy associated with the shedding motion but also altering the structure of the vortex shedding. As such in the following section, Kochin's derivation for the drag due to a vortex street is shown and how coupled changes in the vortex trajectories and circulation affect the drag on a bluff body are presented.

\subsection{Drag Formulation due to the Vortex Street}

To understand the mechanism associated with the increased base pressure, contributions to the drag due to the shed vortices are considered. Following Kochin (1964), in the presence of a semi-infinite vortex street, the contributions to the form drag can be decomposed into three terms as given in Eq. 11. Each of these terms can be estimated from the velocity field obtained with PIV. The contribution to the drag, expressed in non-dimensional form, is:

$$
C_{d}=2\left[\frac{\Gamma_{v s}^{2} f_{v s}}{2 \pi U_{c}}+\Gamma_{v s}(h / D) f_{v s}-\frac{\Gamma_{v s} f_{v s}(h / D)\left(1-U_{c}\right)}{U_{c}}\right],
$$

where $\Gamma_{v s}^{2} f_{v s} / 2 \pi U_{c}$ accounts for the balance of momentum due to the fluid within the domain at an instant, while both $\Gamma_{v s}(h / D) f_{v s}$ and $\Gamma_{v s} f_{v s}(h / D)\left(1-U_{c}\right) / U_{c}$ account for the change in momentum due to the fluid entering and exiting the domain over the period $1 / f_{v s}$ due to the freestream and the motion of vortices respectively. The shedding frequency $f_{v s}$ may be determined directly from the spectra of the velocity fluctuations or POD temporal coefficients (Fig. 4). The circulation of the vortices $\Gamma_{v s}$ is established in Table 9 and the lateral spacing $h / D$ and convective velocity $U_{c}$ are computed from the average trajectories of the vortex centroids over the shedding cycle. The location of the cores are computed using the Q-criterion and second area moment of the $z$-vorticity where $Q>0$ before exiting the PIV domain. The convective velocity pf the vortices is estimated by performing a linear regression of the streamwise position of the centroids versus phase once the vortices reach a constant velocity. The slope $\left(m=\frac{\Delta x / D}{\Delta \theta}\right)$ is then used to calculate an average velocity as $U_{c}=m \times 360 f_{v s}$ and gathered in Table 2 


\begin{tabular}{||c|c|c|c|c|c||}
\hline$N$ & $h / D$ & $\frac{\Delta x / D}{\Delta \theta}$ & $f_{v s}$ & $U_{c}$ & $\Gamma_{v s}$ \\
\hline \hline 0 & 1.020 & 0.01243 & 0.188 & 0.841 & 2.60 \\
\hline 2000 & 0.938 & 0.01039 & 0.194 & 0.726 & 2.19 \\
\hline 2180 & 0.685 & 0.01028 & 0.211 & 0.781 & 2.16 \\
\hline 2200 & 0.892 & 0.01026 & 0.212 & 0.779 & 2.10 \\
\hline 2350 & 1.158 & 0.01096 & 0.208 & 0.821 & 1.96 \\
\hline 3000 & 0.876 & 0.00800 & 0.222 & 0.639 & 1.55 \\
\hline \hline
\end{tabular}

Table 2: Summary of important quantities extracted from the average vortex trajectories

Substituting the values from Table 2 into Kochin's formulation (Eq.(1)) an estimate for the average coefficient $\left(\overline{C_{d}}\right)$ is computed and summarized in Table 3 . The coefficient of drag for the baseline flow $\left(\overline{C_{d}}=1.16\right)$ is in agreement with values from the literature being reported at $\overline{C_{d}}=1.2$ for a square cylinder with rounded leading edges (Heddleson et al., 1957).
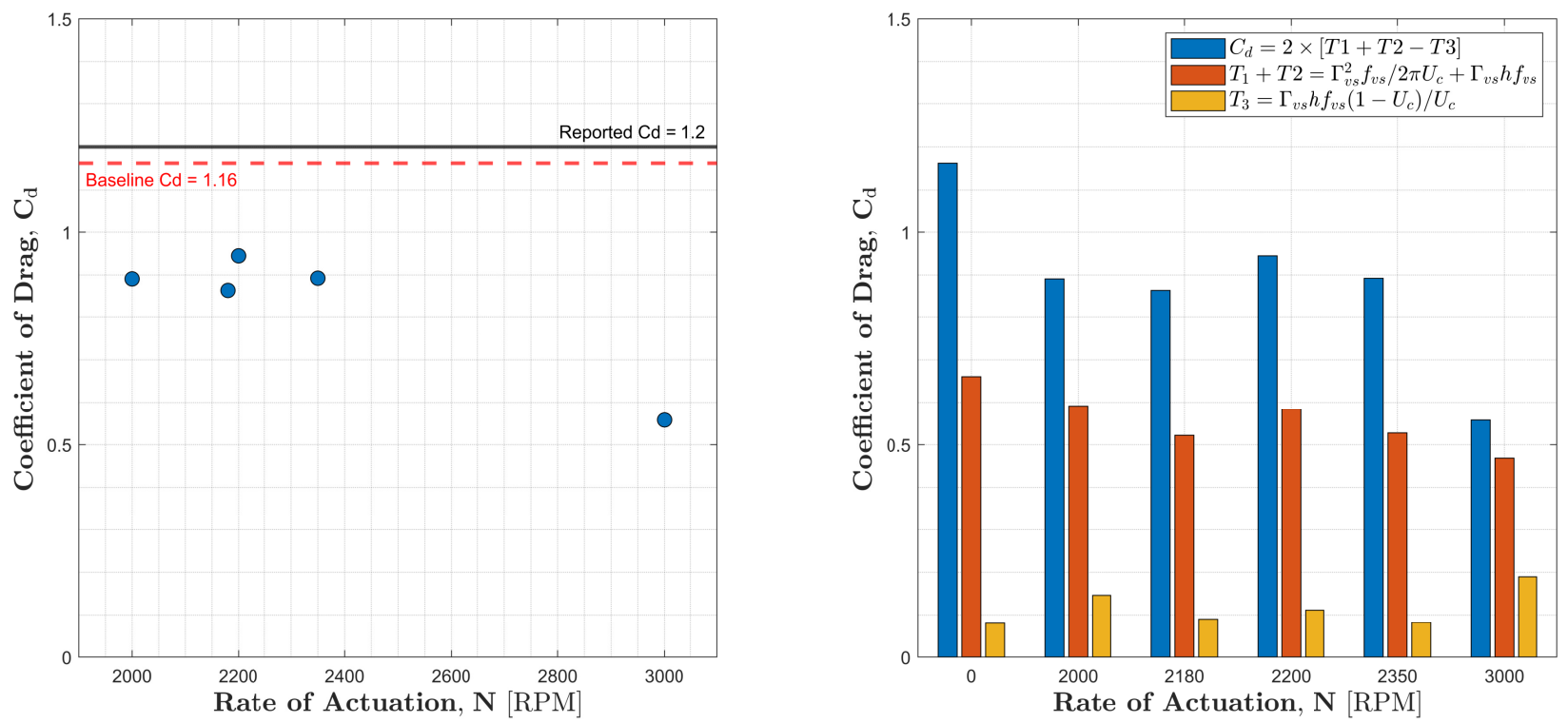

Figure 10: Estimate of the coefficient of drag from PIV measurements and base pressure based on Kochin's formulations for the drag due to a vortex street.

\begin{tabular}{||c|c|c|}
\hline$N$ & $-\overline{C_{p b}}$ & $\overline{C_{d}^{P I V}}$ \\
\hline \hline 0 & 1.299 & 1.162 \\
\hline 2000 & 1.141 & 0.891 \\
\hline 2180 & 1.144 & 0.864 \\
\hline 2200 & 1.121 & 0.945 \\
\hline 2350 & 1.119 & 0.893 \\
\hline 3000 & 1.096 & 0.558 \\
\hline \hline
\end{tabular}

Table 3

The downward trend in estimated $C_{d}$ from Kochin's formulation aligns with the observed increase in the base pressure noted by Figure $3 \mathrm{a}$. It is worth noting that as Kochin's formulation is based on a potential flow solution it does not account for the dissipation due to turbulence and skin friction on the body; as such the slightly under reported values for the baseline are to be expected. Figure 10a shows a significant decrease from the baseline, a plateau while synchronized with the second harmonic of the actuation, followed by another decrease from 2350 to 3000 RPM as the shedding desynchronizes and becomes quasi-periodic. 
To gain insight into the observed changes in drag and base pressure, the terms of the formulation may be separated into two major contributors, balance of projected momentum by the fluid within the domain plus the fluid entering/exiting the field of view over a period $\left(1 / f_{v s}\right)$ as the sum of $T 1+T 2$, and the projected momentum of the fluid leaving the domain due to motion of the vortices, T3. These contributions are shown in Figure $10 \mathrm{p}$. The difference between these two values estimates the drag related to the strength and trajectory of the vortices. From the baseline to actuated cases, $T 1+T 2$ steadily decreases matching the reduced circulation whereas $T 3$ varies over the range with a local maxima at 2000 and 3000 RPM. These maxima match the lowest convective velocities and widest lateral spacing. These changes indicate that as the actuation harmonics shifts the shedding between states of lock-on and quasi-periodicity the mechanism associated with the decreased drag is not simply a reduction of the shed circulation but changes related to the convection rate of the vortices and, in particular, their trajectories.

\section{Conclusions}

The effects of frequency based moving surface actuation were investigated for a square cylinder bluff body wake. It was observed through the POD results that the vortex shedding frequency shifts between the actuation frequency harmonics as the RPM increases, moving between regimes of lock-on and quasi-periodicity. The amount of the TKE and magnitude of the Reynolds stresses associated with shedding were found to decrease as the actuation rate increased. Conversely, the TKE in the entire PIV domain was found to remain relatively constant as actuation increases and therefore the energy associated with shedding is redistributed to other coherent motions. Kochin's formulation for the drag due to an infinite vortex street was evaluated and seen to be in agreement with the trend observed in the base pressure. Differences in the drag formulation as the rate of actuation $N$ increased were isolated to the effect of the vortex impulse as the primary mechanism for drag reduction.

\section{References}

Baek SJ and Sung HJ (2000) Quasi-periodicity in the wake of a rotationally oscillating cylinder. Journal of Fluid Mechanics 408:275-300

Deb K, Pratap A, Agarwal S, and Meyarivan T (2002) A fast and elitist multiobjective genetic algorithm: Nsga-ii. IEEE transactions on evolutionary computation 6:182-197

Heddleson C, Brown D, and Cliffe R (1957) Summary of drag coefficients of various shaped cylinders. Technical report. GENERAL ELECTRIC CO CINCINNATI OH

Kochin NE (1964) Theoretical hydrodynamics. International Publications pages 214-218

Munshi S, Modi V, and Yokomizo T (1997) Aerodynamics and dynamics of rectangular prisms with momentum injection. Journal of Fluids and Structures 11:873-892

Okajima A (1982) Strouhal numbers of rectangular cylinders. Journal of Fluid mechanics 123:379-398

Williamson C (1996) Vortex dynamics in the cylinder wake. Annual Reviews of Fluid Mechanics 28:477539

Wu CJ, Wang L, and Wu JZ (2007) Suppression of the von kármán vortex street behind a circular cylinder by a travelling wave generated by a flexible surface. Journal of Fluid Mechanics 574:365

Xu F, Chen WL, Bai WF, Xiao YQ, and Ou JP (2017) Flow control of the wake vortex street of a circular cylinder by using a traveling wave wall at low reynolds number. Computers and Fluids 145:52-67 